\title{
Summary of Myanmar Clinical Practice Guidelines for Disorders of Thyroid Function
}

\author{
Than Than Aye ${ }^{1}$, Tint Swe Latt ${ }^{2}$, Moe Wint Aung 3 , Ko Ko ${ }^{1}$ \\ ${ }^{1}$ Department of Medicine, North Okkalapa General Hospital, University of Medicine (2), Yangon, Myanmar \\ ${ }^{2}$ University of Medicine (2), Yangon, Myanmar \\ ${ }^{3}$ Department of Medicine, Yangon General Hospital, University of Medicine (1), Yangon, Myanmar
}

\begin{abstract}
Disorders of thyroid function as well goiters are not uncommon in Myanmar, and individuals with these medical problems are frequently seen initially by general practitioners rather than specialists. Hence, guidelines for the recognition, diagnosis, and treatment of thyroid disorders which are developed by a multidisciplinary panel of experts are essential, not so much for standardization of management but to ensure the delivery of excellent, evidence-based, locally applicable, equitable and patient-centered care for people with disorders of thyroid function. This document is a summary of the guidelines for the management of hyperthyroidism and hypothyroidism in the outpatient, as well as for subclinical thyroid disorders, and special circumstances, such as thyroid emergencies (thyroid storm and myxedema coma) and thyroid diseases during pregnancy.
\end{abstract}

Keywords: clinical practice guideline, Myanmar, thyroid disorders

\section{Introduction}

Thyroid disorders, in particular, goiter, thyrotoxicosis, and hypothyroidism are common in daily clinical practice in Myanmar. Patients with goiter are commonly seen in the northern and eastern parts of the country where most of the people live in hilly regions. Goiter is also common in the delta and coastal region of the country since the soil in these regions is usually deficient in iodine content. On the recommendation of the $24^{\text {th }}$ National Health Committee meeting (1997), the Ministry of Mines issued a regulation which stated that all factories should be licensed for production on iodized salt for animal and human consumption in 1999. Universal Salt Iodization (USI) is the major intervention for elimination of iodine deficiency disorders.

\section{Summary of methodology of clinical practice guideline (CPG) development}

In the development of this CPG on thyroid disorders, the following issues are taken into consideration, in that the recommendations must be:

- Evidence-based

- Adapted to the local setting

- Considers patient's values in decision making

- Ensures equity

The aims of the Myanmar CPG on thyroid disorders were: to provide a framework to assist health care professionals in the management of common thyroid disorders, and aid primary care physicians in appropriate referrals to specialists.
The CPG was developed by a multidisciplinary work group consisting of endocrinologists (including pediatric endocrinologists), general physicians (Internists), senior physicians from the Radio Nuclear Department and obstetrician-gynecologists.

This document mainly focused on the diagnosis and management of hyperthyroidism and hypothyroidism.

\section{Hyperthyroidism}

The term "hyperthyroidism" encompasses a heterogeneous group of disorders, all characterized by elevated levels of thyroid hormones in the blood due to hyperfunction of thyroid gland, i.e. increased synthesis and release of thyroid hormones. The term "thyrotoxicosis" refers to a clinical state that results from inappropriately high thyroid hormone action in tissues.

The most common causes in Myanmar include Grave's disease, Toxic Multinodular goiter and Toxic Thyroid nodule. Less common causes include Thyroiditis and Iodine induced Thyrotoxicosis (Jod-Basedow) which may be due to excess iodine given during the National Salt Iodization Program.

\section{Diagnosis}

\section{Assessment of disease severity}

All patients with known or suspected hyperthyroidism should undergo a comprehensive history taking and physical examination which include measurement of pulse rate, blood pressure, respiratory rate, and body weight. In addition, thyroid size; presence or absence of thyroid

Corresponding author: Prof. Tint Swe Latt

President, Myanmar Society of Endocrinology and Metabolism

University of Medicine, Yangon, Myanmar

E-mail address: proftsl@gmail.com 
tenderness, symmetry, and nodularity; pulmonary, cardiac, and neuromuscular function; and presence or absence of peripheral edema, eye signs, or pretibial myxoedema should be assessed.

\section{Biochemical evaluation}

\section{Thyrotoxicosis}

Any clinical suspicion of thyrotoxicosis is confirmed by serum $\mathrm{TSH}_{\text {, free }} \mathrm{T}_{3}$ and $\mathrm{T}_{4}$. A typical biochemical picture of elevated $\mathrm{T}_{4}$ and $\mathrm{T}_{3}\left(\mathrm{FT}_{4}\right.$ and $\mathrm{FT}_{3}$ ) with associated undetectable serum TSH $(<0.1 \mathrm{mU} / \mathrm{L})$ is diagnostic of primary Hyperthyroidism.

\section{T3 Thyrotoxicosis}

It is defined as normal serum $\mathrm{T}_{4}$, serum $\mathrm{TSH}<0.01 \mathrm{mU} / \mathrm{L}$ (or undectable) and elevated serum $\mathrm{T}_{3}$. It may present the earliest stages of the disease or that caused by an autonomously functioning thyroid nodule.

\section{Subclinical hyperthyroidism}

It is defined as a normal serum free $\mathrm{T}_{4}$ estimate and normal total $\mathrm{T}_{3}$ or free $\mathrm{T}_{3}$ estimate, with subnormal serum TSH concentration.

Non-thyroidal illness and drug affecting abnormal thyroid function tests are diagnosed by history, mildly suppressed TSH (0.1-0.4 mU/L) with atypical $\mathrm{T}_{3}$ and $\mathrm{T}_{4}$. e.g., normal or low T3. Re-testing of TFT is advisable once other comorbid condition is eliminated.

If thyroiditis is suspected due to acute onset with fever, pain and tender thyroid swelling, suppressed uptake of radioactive $\mathrm{I}^{131}$ should be demonstrated.

Particular care is required in the diagnosis of hyperthyroidism in patients taking amiodarone and use of this drug should typically be accompanied by periodic evaluation of thyroid function tests.

\section{Treatment plan}

The treatment of Graves' hyperthyroidism includes medical treatment by anti-thyroid drugs, radioactive iodine $\left(\mathrm{I}^{131}\right)$ and thyroid surgery.

Treatment with propanolol, atenolol, metoprolol or other beta-blockers should be used for patients with symptomatic thyrotoxicosis. They are the sole form of treatment required in cases of thyroiditis with mild symptoms.

Choice of treatment is decided by primary care physician with opinion obtaining from specialist after explanation about the risks, benefits, potential side effects, course of the disease according to various forms of treatment to the patients prior to initiation.

\section{Choice of therapy}

\section{Antithyroid drugs}

ATDs: It is generally the first line treatment for Myanmar patients as the availability of $\mathrm{I}^{131}$ is limited. It is recommended for patients with high likelihood of remission (patients, especially females, with mild disease, small goiters, the elderly with co-morbidities, the patients with previously operated or irradiated necks.

I31: It is recommended for individuals with contraindications to ATD use, severe and recurrent cases, co-morbidities and patients with previously operated or externally irradiated necks.

Surgery: Indicated in patients with symptomatic compression or large goiters $(\geq 80 \mathrm{~g})$; when thyroid malignancy is documented or suspected; and patients with moderate to severe active GO.

\section{Antithyroid drugs}

Carbimazole and methimazole are the drugs that are primarily used for the medical management of hyperthyroidism. Propylthiouracil (PTU) is used when there are minor adverse drug reactions to carbimazole which require alternative drugs; during pregnancy especially in first trimester, and during lactation. It is also used during thyroid crisis. Patients should be informed of the side effects of ATD and the necessity of informing the physician promptly if they should develop any reactions, especially fever and pharyngitis, jaundice, and pruritic rash. A differential white blood cell count should be obtained to detect the development of agranulocytosis. Initial daily doses of methimazole generally range from 10 to $40 \mathrm{mg}$, and for propylthiouracil, 100 to $600 \mathrm{mg}$. There is no clear-cut standard for duration of therapy with ATDs, but when used as primary therapy, they are usually given for 6 months to 2 years.

\section{Assessing response to therapy}

Regular measurement of $\mathrm{FT}_{4} / \mathrm{T}_{4}$ and $\mathrm{TSH}$ is essential in order to adjust drug doses. Measurement of serum TSH alone is not adequate since TSH may remain suppressed for weeks or months. In most cases the $\mathrm{FT}_{4}$ result will be the marker of choice to guide therapy.

Thyroid function tests should be performed every 4-6 weeks initially and reduced to every 3 months once a maintenance dose is achieved.

\section{$\underline{I^{131} \text { therapy }}$}

It is safe with the principal side-effect being the early or late development of hypothyroidism, necessitating lifelong thyroid hormone replacement following $\mathrm{I}^{131}$ treatment.It is contraindicated during pregnancy and breast feeding. As $\mathrm{I}^{131}$ therapy is less available in Myanmar, it is particularly used for severe and recurrent thyrotoxicosis after ATD or those who develop side-effects 
using ATD. Severe cases and those with cardiovascular complications need to be pretreated with ATD to avoid the exacerbation of hyperthyroidism due to $\mathrm{I}^{131}$ induced thyroiditis. In some patients, ATDs may be required for control for several months following radioiodine therapy.

\section{Assessing response to therapy}

Serum FT4 and TSH should be measured in all patients treated with radioiodine. In most cases the FT4 result will be the marker of choice to guide therapy. Thyroid function tests should be performed every 4-6 weeks for at least six months following radioiodine therapy. It may be reduced when the $\mathrm{FT}_{4}$ remains within the reference range, although an annual TFT is still required.

A serum TSH result of $>20 \mathrm{mU} / \mathrm{L}$ following radioiodine therapy should trigger thyroxine therapy.

\section{Surgery}

Whenever possible, patients with GD undergoing thyroidectomy should be rendered euthyroid with carbimazole/methimazole. Potassium iodide should be given in the immediate preoperative period. When the patient is allergic to antithyroid medication, the patient should be adequately treated with beta-blockade and potassium iodide in the immediate preoperative period. Patients must be cautioned about potential complications of surgery.

Following thyroidectomy, serum calcium or intact parathyroid hormone levels should be measured, and that oral calcium and calcitriol supplementation be administered based on these results.

Following thyroidectomy for GD, L-thyroxine should be started at a daily dose appropriate for the patient's weight $(0.8 \mathrm{mg} / \mathrm{lb}$ or $1.7 \mathrm{mg} / \mathrm{kg})$, and serum TSH measured $6-8$ weeks postoperatively.

\section{Long-Term Follow Up}

All patients who have received radioiodine therapy for hyperthyroidism or surgery (partial thyroidectomy) require life-long follow-up to identify development of hypothyroidism.

TSH plus $\mathrm{FT}_{4}$ should be monitored approximately every 12 months.

Measurement of serum TSH is the most sensitive and specific indicator of onset of hypothyroidism. It can also detect the recurrence of hyperthyroidism.

\section{Subclinical Hyperthyroidism}

Subclinical hyperthyroidism is defined as a low serum $\mathrm{TSH}$ in the presence of normal concentrations of $\mathrm{FT}_{4}$ and $\mathrm{FT}_{3}$. It is essentially a biochemical diagnosis and clinical signs and symptoms are absent or mild/non-specific.
Patients with subclinical hyperthyroidism that cannot be explained by non-thyroidal illness or drug therapy should have repeat thyroid function testing (TSH with serum $\mathrm{FT}_{4}$ and $\mathrm{FT}_{3}$ ).

Growing evidence suggests subclinical hyperthyroidism in elderly subjects is associated with increased risk of development of atrial fibrillation and increased vascular mortality. Persistent subclinical hyperthyroidism should prompt specialist referral.

Untreated subclinical hyperthyroidism should be followed into the long term by testing thyroid function every 6-12 months.

\section{Special problems}

\section{Hyperthyroidism and Pregnancy}

Pregnancy may be adversely affected by poorly controlled hyperthyroidism, with an increased rate of fetal loss. The diagnosis of hyperthyroidism in pregnancy should be made using serum TSH values, and either total $\mathrm{T}_{4}$ and $\mathrm{T}_{3}$ with total $\mathrm{T}_{4}$ and $\mathrm{T}_{3}$ reference range adjusted at 1.5 times the non-pregnant range or free $\mathrm{T}_{4}$ and free $\mathrm{T}_{3}$ estimations with trimesterspecific normal reference ranges. Transient hCG-mediated thyrotropin suppression in early pregnancy should not be treated with antithyroid drug therapy.

The goal of treatment during pregnancy is to maintain euthyroidism, using the smallest doses of ATDs possible. Propylthiouracil is preferred in pregnancy especially during first trimester because it crosses the placenta less than methimazole. Methimazole can be reused after $1^{\text {st }}$ trimester.

Hyperthyroid pregnant patients should be seen at 4- to 6week intervals. Thyroid function should be assessed monthly, and the antithyroid drug dose adjusted as required. Total T4 and T3 values should be kept at the upper normal range with suppressed TSH.

Patients treated for hyperthyroidism during pregnancy should be reevaluated 6 weeks post partum.

\section{Management of thyroid storm}

When the diagnosis is suspected clinically, therapy must be initiated immediately. Treatment should be initiated in the intensive care unit. (1) (propylthiouracil or methimazole); (2) potassium iodide, ipodate); and (3), corticosteroids. Because of the complexity of thyroid storm, it is recommended that an endocrinologist participate in the evaluation and management of such patients. 


\section{Hypothyroidism}

Treatment guidelines for patients with hypothyroidism Hypothyroidism is a disorder of diverse causes in which the thyroid gland fails to secrete adequate amounts of thyroid hormone. Majority of cases are due to radioiodine therapy, surgery and chronic autoimmune thyroiditis (Hashimoto's or spontaneous atrophic)

The diagnosis of primary hypothyroidism requires the measurement of both TSH and FT4 .Subjects with a TSH of $>10 \mathrm{mU} / \mathrm{L}$ and FT4 below the reference range have overt primary hypothyroidism and should be treated with thyroid hormone replacement.

When autoimmune thyroiditis is the suspected underlying cause, it is helpful to confirm antithyroid antibody titers, either antimicrosomal antibody (anti-TPO antibody) or anti-thyroglobulin antibody.

\section{Treatment plan}

Levothyroxine sodium is the treatment of choice for the routine management of hypothyroidism. The recommended approach in primary hypothyroidism is to titrate thyroxine therapy against the TSH concentration whilst assessing clinical well-being. The target is a serum TSH within the reference range.

In the majority of patients $50-100 \mu \mathrm{g}$ thyroxine can be used as the starting dose, although a higher starting dose may be indicated in patients post-thyroidectomy. Thereafter, alteration of the dose is achieved by using $25-50 \mu \mathrm{g}$ increments and repeat measurement of TSH 2-3 months after a change in dose.

In elderly patients and patients with with ischaemic heart disease consideration should be given to commence replacement with $25 \mu \mathrm{g}$ thyroxine and increase the dose in $25 \mu \mathrm{g}$ increments in an attempt to avoid cardiac complications.

Thyroxine should not be administered together with sucralfate, aluminium hydroxide, ferrous sulphate, calcium carbonate which can delay the absorption.

Once the TSH concentration has been normalized, the need for frequent visits is reduced. Visit frequency of every 6 to 12 months is then sufficient, depending on the clinical situation. Should it be necessary to adjust a patient's dosage, he or she should return in 2 to 3 months to assess the therapeutic response and to remeasure the TSH concentration.

A TSH concentration should be measured at least annually. For patients who have recently started receiving levothyroxine or who have had their dosage, type, or brand of thyroid preparation changed, the TSH concentration should be measured after 8 to 12 weeks.

\section{Special problems}

\section{Pregnancy}

During pregnancy, many hypothyroid patients have an increase in levothyroxine requirement, which can be detected with a TSH measurement. The patient should be checked during each trimester to make sure that the TSH concentration is still normal, with further adjustments as indicated by the appropriate testing. The levothyroxine dose should return to the pre-pregnancy dose immediately after delivery and a serum TSH level should be obtained 6 to 8 weeks post partum.

\section{Subclinical Hypothyroidism}

Subclinical (mild) hypothyroidism is characterized by a TSH above the reference range with a FT4 measurement within the reference range. It should be confirmed by repeat thyroid function testing 3-6 months after the original result.

Therapy for subclinical hypothyroidism is probably advisable, especially if thyroid autoantibodies are positive, because overt hypothyroidism develops with high frequency in such patients. If not, they should be evaluated at yearly intervals.

\section{Myxoedema coma}

Coma caused by myxedema is a rare, life-threatening state in which severe, usually long-standing hypothyroidism markedly worsens. In general, it occurs in elderly individuals and is usually precipitated by an intercurrent medical illness. The clinical manifestations, in addition to obtundation or coma, may include hypothermia, bradycardia, respiratory failure, and even cardiovascular collapse.

Therapy of myxoedema coma includes intravenous administration of levothyroxine and/or liothyronine sodium as well as pharmacologic doses of glucocorticoids. Also, precipitating or associated disorders must be aggressively treated.

Patients with myxoedema coma should be treated in the intensive care unit with appropriate monitoring and with the participation of an endocrinologist.

\section{Post-partum thyroiditis}

Post-partum thyroid dysfunction is characterized as thyrotoxic phase occurring in the first 3 months post partum, followed by hypothyroid phase that occurs 3-6 months after delivery, followed by spontaneous recovery.

The thyroid is painless and often there is no goiter; symptoms are often non-specific (e.g., tiredness, anxiety, depression), so awareness and use of TFT are important. $\mathrm{TPOAb}$ results are usually positive, and the presence of $\mathrm{TPO}-\mathrm{Ab}$ in early pregnancy predicts a $30-50 \%$ chance of postpartum thyroiditis developing. 


\section{Screening for thyroid disease during pregnancy}

Pregnant women with type-1 diabetes, previous history of thyroid disease, current thyroid disease, family history of thyroid disease, goiter, and symptoms of hypothyroidism should have thyroid function assessment either at diagnosis or at antenatal booking, or even before conception if feasible.

If the thyrotoxicosis is secondary to postpartum thyroiditis treatment is not required but the TFT should be monitored to detect onset of hypothyroidism.

Further tests (Thyroid antibody tests) are required to differentiate postpartum thyroiditis from Graves' disease

If the thyroid function tests show hypothyroidism thyroxine therapy should be started in a symptomatic patient.

\section{Neonatal screening for congenital hypothyroidism}

All newborn babies should be screened for congenital hypothyroidism by measurement of bloodspot TSH using a sample collected within 2-7 days after birth, as part of a national screening programme.

The measurement of thyroid related hormones as part of a neonatal screening programme should be restricted to specialist laboratories and should have a turnaround time of $<5$ days.

Confirmation of the diagnosis of congenital hypothyroidism involves measurement of serum TSH and $\mathrm{FT}_{4}$ in both mother and neonate and $\mathrm{TSH}$ receptor antibody in the mother.

All hypothyroid neonates should be treated as early as possible. Treatment must be started within the first 18 days of life.

\section{Iodine-induced hyperthyroidism}

Iodine-induced hyperthyroidism is usually occurred in patients with underlying thyroid autonomy, especially those living in areas with mild-to-moderate iodine deficiency. Common sources of iodine excess are iodized salt, amiodarone and radiocontrast material. High iodine intake may also be followed by relapse of hyperthyroidism in patients with previous GD who are in remission after ATD therapy.

Iodine-induced hyperthyroidism (the Jod-Basedow phenomenon) is usually self-limited, lasting 1-18 months. Treatment includes avoidance of additional iodine and administration of beta-blockers alone or with ATDs. Radioactive iodine is not an option until the iodine load has been cleared. Surgery may be used in patients allergic or resistant to antithyroid drugs.
All patients on amiodarone therapy should have thyroid function tested before commencing treatment and then should be routinely monitored every 6 months thereafter whilst on treatment and up to 12 months after cessation of therapy.

\section{References}

1. UK Guidelines for the use of thyroid function test. Association of Clinical Biochemistry, British Thyroid Association, British Thyroid Foundation. www.british-thyroid-association.org/TFT_guideline_ final_version_July_2006.pdf

2. American Association of Clinical Endocrinologist Medical Guidelines for Clinical Practice for the evaluation and treatment of Hyperthyroidism and Hypothyroidism. 2011. Endocr Prac Vol 17.

3. Vaidya B, Pearce S H S. Management of Hypothyroidism in Adults. BMJ 2008; 337:284-289.

4. Woeber K A. Update on the management of Hyperthyroidism and Hypothyroidism. Arch Intern Med.2000;160:1067-1071.

Requests for further details on the recommendations shall be forwarded to the Myanmar Society of Endocrinology and Metabolism (MSEM). 